Knee extensor fatigue resistance of young and older men and women performing sustained and brief intermittent isometric contractions

Running Title: Knee extensor fatigue

Authors: Jamie S. McPhee (PhD) ${ }^{1}$; Thomas M. Maden-Wilkinson (BSc) ${ }^{2}$; Marco V. Narici $(\mathrm{PhD})^{3}$; David A. Jones $(\mathrm{PhD})^{1} \&$ Hans Degens $(\mathrm{PhD})^{1}$

${ }^{1}$ School of Healthcare Science, Manchester Metropolitan University, Manchester, UK ${ }^{2}$ School of Sport, Exercise and Health Sciences. Loughborough University, UK.

${ }^{3}$ University of Nottingham, School of Graduate Entry Medicine and Health, Derby Royal Hospital, Uttoxeter Road, Derby, UK

This study was supported by funding from EU FP7 'MYOAGE' (nr. 223576)

Corresponding Author:

Jamie S. McPhee, School of Healthcare Science, Manchester Metropolitan University, John Dalton Building, Manchester, M1 5GD, UK

Email: j.s.mcphee@mmu.ac.uk 


\title{
Knee extensor fatigue resistance of young and older men and women performing sustained and brief intermittent isometric contractions
}

\begin{abstract}
Introduction: Susceptibility to muscle fatigue during aging could depend on muscle activation patterns. Methods: Young (mean age 22 yrs) and older (mean age 70 yrs) men and women completed two fatigue tests of knee extensor muscles using voluntary and electrically stimulated contractions. Results: Older subjects displayed a shift to the left of the torque-frequency relationship and held a sustained voluntary isometric contraction at 50\% maximal strength for significantly longer than young $(P<0.001)$. Young and old showed similar fatigue during electrically-induced, intermittent isometric contractions (1-s on, 1-s off for $2 \mathrm{~min}$ ), but women fatigued less than men $(P=0.001)$. Stronger muscles fatigued more quickly, and slower contractile properties were associated with longer sustained contractions. Discussion: The slowing and weakness of older muscle was associated with superior fatigue resistance during sustained isometric contractions. Young and old showed similar fatigue following a series of brief, intermittent contractions, but women fatigued less than men.
\end{abstract}

Key words

Skeletal muscle; fatigue; aging; weakness; Myoage 
List of Acronyms and Abbreviations

YM: young men

YW: young women

OM: older men

OW: older women

MVC: maximal voluntary isometric contraction torque

T: the amplitude of the superimposed doublet

T: the value of the resting doublet

ANOVA: Analysis of variance

SD: Standard deviation

SEM: Standard error of the mean

ATP: Adenosine triphosphate 


\section{INTRODUCTION}

Older people have been shown to have greater fatigue resistance during sustained voluntary or electrically-stimulated isometric contractions of upper limb or lower leg muscles ${ }^{1-5}$. During sustained contractions where the intensity is greater than around $40 \%$ maximal isometric strength the blood supply to the active muscle becomes occluded by the pressure generated within the muscle ${ }^{6}$ and this can restrict oxidative energetic recovery. The rate of fatigue in this instance is due mainly to the rate of energy utilization. For oxidative recovery to occur there needs to be a period of relaxation between contractions. Typical patterns of muscle activation usually involve intermittent contraction/relaxation cycles, allowing blood to perfuse the muscle during the relaxation phase. Thus, the rate of fatigue that occurs following intermittent muscle activation reflects the balance between energy expenditure during contraction and regeneration during relaxation. Some studies have reported less fatigable muscles in older compared with young adults when performing intermittent contractions ${ }^{7-11}$. However, all of these studies utilized relatively long contraction durations $(\sim 5-30-\mathrm{s}$ contractions with similar rest interval), and these studies were carried out in peripheral muscles acting on the ankle joint or hand.

Studies of the quadriceps femoris have provided conflicting reports which might be explained by methodological differences in contraction/relaxation protocols, the use of voluntary or electrically stimulated contractions, and possible differences between the sexes. Callahan et $\mathrm{al}^{12}$ found that older men and women were more fatigue resistant when they performed repeated intermittent, voluntary 5-s contraction / 5-s recovery cycles. However, in other studies that utilized a recovery time between contractions of $\leq$ 2-s, no difference was seen in fatigue profiles of young and older men ${ }^{13-15}$. It is unclear 
whether age-related differences in fatigue would be seen if the contraction and relaxation durations were both reduced to $\leq 2$-s. It also remains to be determined whether sex differences similar to those seen in young individuals following intermittent fatiguing contractions ${ }^{16}$ are also present in old, or whether such differences diminish, as was the case for sustained contractions of the arm flexors ${ }^{3}$.

Quadriceps muscle fatigue following intermittent activation has been studied using voluntary $^{12,13}$ as well as electrically-stimulated activation ${ }^{14-16}$. Electrical stimulation protocols have the advantage that they bypass possible bias related to central activation of muscle, such as the possibility that older people show greater decline in central activation 17,13 or a different response of the motor unit firing rate during fatiguing contractions $^{11}$. They therefore provide a greater focus on factors at or distal to the neuromuscular junction and in the muscle itself as causes of fatigue. Stimulated contractions have also been used to study age-related differences in fatigue following prolonged, sustained contractions of small peripheral muscles ${ }^{4}$. Sustained stimulated contraction of the quadriceps can be very unpleasant and cause muscle soreness lasting several days (unpublished observations). Therefore, to distinguish the effects of voluntary activation failure or motivation from peripheral muscle fatigue during a sustained contraction of quadriceps, brief electrically stimulated-contractions can be applied on to sustained voluntary activation ${ }^{18}$.

In light of the questions raised here, this study was undertaken with the objective to examine age and sex differences in fatigue resistance following two different types of contractile activity, one a sustained voluntary isometric contraction to task-failure and the other an intermittent series of brief electically-stimulated contractions. 


\section{MATERIALS AND METHODS}

\section{Subjects and ethical approval}

The study conformed to the latest revisions of the Declaration of Helsinki and received approval from the ethics committee of the Faculty of Science and Engineering at Manchester Metropolitan University. Data were collected from 9 young men (YM), 8 young women (YW), 9 older men (OM), and 10 older women (OW), who were participating in the MYOAGE Study ${ }^{19}$. All subjects provided written informed consent after the procedures had been explained fully. Young subjects were recruited from among the university student population, and older subjects were recruited from the local community. Subject characteristics are shown in Table 1. All were healthy and recreationally active, but none were engaged in sporting competitions. Subjects were excluded if they had a history of neuromuscular or skeletal disease or injury, cardiovascular, metabolic or other major disease, or if they were suffering pain or injury that would prevent them from performing maximal effort contractions of the leg muscles. Older subjects received clearance from their medical doctor to take part in the study.

\section{Overview of study procedures}

Subjects completed 2 fatigue protocols, 1 on each leg. All assessments were of the knee extensor muscles using a custom built isometric testing dynamometer with torque signals recorded via a computer interface running customized Labview (National Instruments Corporation, Texas, USA) and Matlab software (Matlab, the Mathwork Inc., S Natik, MA, USA). The leg and fatigue protocol to be used first were chosen at random. On the first leg, subjects completed assessments of maximal voluntary isometric contraction torque (MVC) with assessment of the voluntary activation capacity using 
the interpolated twitch technique ${ }^{18}$. After a short rest of around $2 \mathrm{~min}$, contractile properties were assessed using a series of electrically stimulated contractions ${ }^{16}$. After another short rest period, the first fatigue test was performed. Subjects were then allowed to rest for around 6 min while the equipment was set up to test the second leg. On the second leg, MVC was assessed, and after a short rest of around 2 min, the second fatigue test was completed. No subject reported pain (e.g. in the knee) that interfered with the ability to perform contractions, and the protocols were well tolerated.

\section{Isometric maximal voluntary contraction torque}

Knee extensor MVC was assessed at a knee angle of $90^{\circ}$ (where full extension is $0^{\circ}$ ) and a back support at $85^{\circ}$ (where lying supine is $0^{\circ}$ ). A strap was secured firmly across the hip joint, and the lower leg was secured firmly to the force transducer $2 \mathrm{~cm}$ above the ankle malleolus. Subjects were familiarized with knee extension exercise by performing

3 contractions at around $50 \%$ of maximal effort, each lasting $3 \mathrm{~s}$ and another 2 contractions at around $80 \%$ maximal effort. After 2 min rest, subjects were instructed to perform a maximal effort by increasing force rapidly and sustaining the maximal effort for around $3 \mathrm{~s}$. This maximal effort was repeated twice more or until the highest 2 values were within $10 \%$, the highest value being taken as MVC. Visual feedback was available throughout, and strong verbal encouragement was provided.

\section{Voluntary activation}

The capacity to activate the knee extensor muscles during isometric contractions was assessed using the interpolated twitch technique ${ }^{18,20}$. The procedure was fully explained, and the subjects were slowly accustomed to the electrical stimulation. Muscle stimulation electrodes (AmericanImex, CA, USA) were placed over the proximal (anode) and distal (cathode) heads of the quadriceps femoris muscles. The electrodes were 
connected to a Digitimer DS7AH (Digitimer Ltd, Herts, UK) via anode and cathode leads. Voltage was set at $400 \mathrm{~V}$ at a pulse width of $200 \mu \mathrm{s}$. A "doublet" stimulus (2 pulses separated by $10 \mathrm{~ms}$ ) that induced $\geq 30 \%$ MVC was applied to the relaxed muscle. Approximately 1-s later the subject performed a maximal voluntary effort, and a doublet was applied again at the highest point of the MVC. This test was performed twice, and the percentage voluntary activation was calculated as:

$$
\text { Voluntary activation }=100 *(1-\mathrm{t} / \mathrm{T})
$$

where $t$ is the amplitude of the superimposed doublet (i.e. the size of the additional peak) and $\mathrm{T}$ the value of the resting doublet.

\section{Torque-Frequency relationship and rates of contraction and relaxation}

The torque-frequency relationship was determined using pre-set pulse width, voltage, and current $(200 \mu \mathrm{s}, 400 \mathrm{~V}$, and the current, which differed between subjects, that elicited 25\% MVC at $30 \mathrm{~Hz})$. The muscle was stimulated at 7 different frequencies $(1,10$, $15,20,30,50$, and $100 \mathrm{~Hz}$ ) in random order, separated by $60-\mathrm{s}$, and with the tetani lasting 2-s. The maximal torque output at each frequency was recorded and expressed as a percentage of torque elicited at $100 \mathrm{~Hz}$. Relaxation rate (delta force/delta time), normalized relaxation rate (relaxation rate/peak force), and half relaxation time of the $30 \mathrm{~Hz}$ contraction were also calculated ${ }^{16}$.

\section{Sustained Fatigue test}

The procedure for the sustained isometric contraction fatigue test was explained fully to subjects. A computer interface was set to show the target force at $50 \% \mathrm{MVC}$, and the subject was allowed to practice holding the torque at the target line for $3-5 \mathrm{~s}$. After a 2 min rest the subject was asked to perform a voluntary contraction at 50\% MVC for as 
long as possible. The end point was taken as the time at which the torque fell below 45\% MVC for more than 3-s despite strong verbal encouragement to regain the required $50 \%$ target. The end point of this fatigue test may be determined by central or peripheral muscle fatigue. Thus, to assess the extent of central activation, stimulated doublets (that elicited around 30\% MVC in rested muscle) were superimposed at 10-s intervals throughout the test in a subgroup of 13 young and 16 older subjects (similar split for men and women). The superimposed stimuli were normalized to a doublet applied to the resting muscle prior to commencement of the test, as described for the calculation of voluntary activation, above. Figure 1 shows an example of raw data in a young and older man.

\section{Electrically stimulated intermittent contraction fatigue test}

The procedures of this test were fully explained to the subject, emphasising the need to remain relaxed throughout the test, avoiding any voluntary contractions of muscles acting around the knee. The test was carried out after setting pulse width, voltage and current at: $200 \mu \mathrm{s}, 400 \mathrm{~V}$ and current that elicited around 25\% MVC at $30 \mathrm{~Hz}$, respectively. A total of 60 stimuli of 1-s duration were applied, each separated by 1-s rest interval over the course of two minutes. Subjects received visual and verbal feedback throughout the test which helped them stay relaxed. This intermittent, stimulated protocol allowed relaxation and hence metabolic recovery, between contractions and bypassed the possible complications of motivation and differences in voluntary activation. The test was well tolerated; all subjects remained relaxed (i.e. no additional voluntary contraction), and all completed the full $2 \mathrm{~min}$ protocol.

\section{Statistical analysis}


Data were analyzed using SPSS v18 (IBM, USA). A two-way ANOVA was used to assess sex and age effects and possible interactions between sex and age. An age x sex interaction would indicate that the effects of age differ between men and women. The Pearson product moment correlation coefficient was used to determine the relationships between variables. Descriptive data in Table 1 are expressed as mean \pm standard deviation (SD), while all other data relating to comparisons between groups are expressed as mean \pm standard error of the mean (SEM). Differences between groups were considered significant at $P<0.05$.

\section{RESULTS}

The older subjects were weaker than young, and women were weaker than men (Table 2; $P<0.001)$. The young group had slightly higher voluntary activation than the older group during maximal isometric knee extension $(P=0.033$; Table 2$)$.

\section{Muscle contractile properties}

Significant sex and age differences were found in rates of relaxation following a brief 30Hz stimulus, with men having faster relaxation than women, and young faster than old (Table 2). The torque/frequency relationships (Figure 2) show that older subjects produced relatively more force than young at $1 \mathrm{~Hz}(P=0.017), 10 \mathrm{~Hz}(P=0.005), 15 \mathrm{~Hz}$ $(P=0.007)$, and $20 \mathrm{~Hz}(P=0.028)$. The torque/frequency relationships did not differ between men and women.

\section{Sustained Contraction Fatigue Test}

Figure 3 shows individual data points for the time to task failure as a function of age in men and women. There was no significant difference between sexes $(P=0.416)$, but the 
older subjects were able to sustain the contraction on average for around 20-s longer than the young subjects (71.2 \pm 5 vs $91.5 \pm 5$ s in young and old, respectively; $P=0.001$ ). Voluntary activation was assessed throughout the fatigue test by using the superimposed doublet stimulation, and the results (Figure 4) show that at the start of the contraction activation was close to $50 \%$, as expected, since the target was set at $50 \%$ MVC. There was then a steady increase in activation until at task failure the older subjects were activating their muscles to approximately $84 \%$ and the young to $88 \%$ of their maximum, with no difference between ages $(P=0.181)$ or sexes $(P=0.908)$ at task failure.

When analyzing the full dataset, time to task failure was significantly related inversely with MVC ( $\mathrm{r}=-0.458 ; P=0.005 ;$ Figure 5$)$ and related positively to $1 / 2$ relaxation time ( $\mathrm{r}$ $=0.360, P=0.034)$.

\section{Intermittent contraction fatigue test}

Intermittent electrically-induced $30-\mathrm{Hz}$ tetani of 1-s duration and 1-s recovery between stimuli were applied to knee extensors to elicit 60 cycles of contraction / relaxation, taking a total of $2 \mathrm{~min}$. The $30-\mathrm{Hz}$ stimulus applied to fresh muscle gave similar relative force in all groups, as can be seen in the torque-frequency relationship (Figure 2).

Figures $6 \mathrm{a}$ and $6 \mathrm{~b}$ show the mean (SEM) data for men and women, respectively, for the full duration of the intermittent fatiguing protocol. Young and old showed similar force loss through the duration of the test $(P=0.480)$, but women fatigued less than men $(P=0.001)$. At the end of the test, the percentage force that remained (fatigue index) was $54.8 \%( \pm 2.2), 56.4 \%( \pm 2.6), 67.8 \%( \pm 3.3)$, and $63.4 \%( \pm 2.7)$ in young men, older men, young women, and older women, respectively. 
When analyzing the full dataset, the fatigue index was correlated inversely with the MVC $(\mathrm{r}=-0.394, P=0.016)$, but not with the $1 / 2$ relaxation time $(\mathrm{r}=0.290, P=0.086)$. The fatigue index from the intermittent stimulated test was not correlated significantly with the time to failure of the sustained contraction test $(r=0.222, P=0.192)$.

\section{DISCUSSION}

We have utilized 2 different fatigue tests to differently stress the mechanisms of energy expenditure and regeneration to examine age and sex differences in skeletal muscle fatigue resistance. The results show that older men and women were able to hold a sustained contraction at 50\% of MVC for around 20 s longer than young men and women. However, when the pattern of activation was changed to short, intermittent contractions with brief rest intervals, the young and older adults showed similar declines in force throughout a series of 60 contractions. The following discussion considers the contrasting findings in the two different fatigue tests as well as sex differences that were evident in the intermittent fatigue test.

\section{Effects of aging on the sustained isometric contraction}

Our results show a longer endurance time to task failure in older compared with young adults during a sustained isometric contraction of the knee extensors, which extends previous work that looked at smaller muscle groups ${ }^{1-5}$. A sustained isometric contraction is a task that involves high voluntary effort, and maintaining the contraction at 50\% MVC becomes increasingly unpleasant, possibly due to build-up of ischemic pain. One possible explanation for the lesser performance of the young subjects is that they were more susceptible to central fatigue than the older subjects. However the use of superimposed stimulation demonstrated that at the point of task failure both young and older subjects were recruiting similar relative fractions of the muscle ( 84 and $88 \%$, 
respectively), indicating that the difference in fatigability reflects mainly differences in peripheral mechanisms of fatigue. Progressive recruitment of motor units and a small contribution of central fatigue at task failure in young and older adults has been reported in previous studies of elbow flexor ${ }^{1,3}$ and plantar flexor muscles ${ }^{17}$.

In all groups (young, old, men, and women) there was approximately a two-fold interindividual variation in time to task failure. One possible factor that has been discussed in relation to individual differences in muscle fatigue is that larger and stronger muscle might fatigue more rapidly in both sustained ${ }^{3}$ and intermittent contractions ${ }^{8,21,10}$. We also observed an inverse relationship between time to task failure and muscle strength (Figure 5). One might think that larger muscles might develop higher intramuscular pressures, causing greater occlusion of blood flow. However, this is unlikely to be the explanation for differences in fatigability between young and old, since oxygenation of a muscle depends on the relative, rather than the absolute, force the muscle generates, ${ }^{6}$ and even relatively small rabbit muscles produce intramuscular pressures sufficient to occlude blood flow ${ }^{22}$.

A reason for the superior performance of older subjects during the sustained isometric contraction probably lies in the different contractile properties of old and young muscle. Slow contracting type I fibers constitute a greater proportion of the overall muscle in older compared with young subjects ${ }^{23}$. Our examination of contractile properties showed muscles of young men to be fastest, followed by young women, with older men and women being the slowest, based on measurements of relaxation rates (Table 2) and the effect this has on the torque/frequency relationship (Figure 2). Slower muscles of older people have been shown to have a lower energy turnover during prolonged isometric contraction and accumulate metabolites at a slower rate $24,8,25$. The lower rate 
of energy turnover of older muscle could be due to slower cross bridge kinetics or lower cation pump activity in the sarcoplasmic reticulum and plasma membranes ${ }^{26}$.

\section{Effects of age on the intermittent contraction fatigue test}

The intermittent fatigue protocol reflects the balance between energy expenditure during contraction and regeneration during relaxation. While older muscles had a longer time to task failure during a sustained isometric contraction, there was no difference between young and older subjects during a series of brief intermittent isometric contractions. This is consistent with 2 recent systematic reviews ${ }^{27,28}$ which suggested that older subjects have superior fatigue resistance during sustained isometric contractions, but this advantage is not seen when performing dynamic (intermittent) contractions. It has been suggested that electrically-evoked muscle contractions may preferentially activate larger, faster fibers and possibly induce greater glycolytic flux that increases the rate of fatigue (for review, see ${ }^{29}$ ). It would then follow that older and young muscle may have differential responses to electrical stimulation due to the higher susceptibility of type 2 fibers to atrophy with aging ${ }^{30}$. However, recent reviews suggest muscle electrical stimulation activates the mix of both fast and slow fibers located within 'range' of the electrodes ${ }^{29,31}$. A more likely explanation is that the ability to regenerate ATP during the rest intervals plays a significant role in delaying the onset of fatigue. Some studies suggest older people have higher oxidative ATP production compared with young8,25. Lower mitochondrial enzyme activity was found in older gastrocnemius but not vastus lateralis muscle ${ }^{32}$. Several other studies show lower oxidative capacity ${ }^{33}$ and mitochondrial content or activity as well as microvascular supply in older muscle compared with young ${ }^{33-38}$. A decrease in oxidative capacity may impair the performance of older muscle during intermittent contractions. 
In contrast, such a decrease in oxidative capacity would play a lesser role in the rate of fatigue during sustained isometric contractions where blood flow is occluded ${ }^{6}$.

Another explanation for the failure of slower, older muscle to translate to greater fatigue resistance during brief intermittent contractions might relate to the energetics of muscle contraction. The energy consumed by a muscle that is shortening and performing work is greater than when it is contracting isometrically (Fenn effect) ${ }^{39}$. Furthermore, the differences between the energy demands of isometric and shortening contractions varies between fast and slow muscles. Barclay et $\mathrm{al}^{40}$ showed that slow mouse soleus muscles had relatively low rates of energy turnover during isometric contractions, but this increased up to four-fold during rapid shortening. In contrast, the fast extensor digitorum longus muscle had higher energy turnover in the isometric state, but this increased only modestly during shortening contractions. Thus, the efficiency of slow muscles decreases to a greater extent than that of fast muscle as the velocity of shortening increases. Extrapolating this to relatively slow older human muscles and faster young muscles, we may assume that, during sustained isometric contractions the lower energy turnover of the older muscles conveys an advantage and permits superior endurance during prolonged contractions, as discussed above. However, during shortening contractions, the energetic demands rise considerably for the slower older muscles, and any advantage they had in the isometric state is lost. Although the brief intermittent contractions we have used are described as "isometric", at the start of each contraction there is considerable shortening as the muscle stretches compliant structures such as tendons, connective tissue, joint structures, and parts of the apparatus. Thus, in addition to developing tension, internal work is also done. This additional energy cost can be considerable, as the energy costs of a series of short 
isometric contractions is nearly twice that of a continuous isometric contraction of the same duration and results in greater force loss and slowing of the muscle ${ }^{41}$.

The energy cost of a single isometric contraction will consist of a fast component at the start of the contraction where internal work is being done and a slower component when the muscle has reached the isometric phase. The proportions of these 2 components will depend on the duration of the contraction. For short contractions of 1 sec, as in this study, the shortening phase might occupy one-third to one-half of the total contraction time and so contribute a great deal to the total turnover, offsetting the advantage that older muscle has during purely isometric contractions. As the duration of the contraction increases, so the relative contribution of the energy associated with the initial shortening will diminish, and slower muscle will regain its energetic "advantage". This may explain why several studies that utilized intermittent isometric contractions of 5- up to 30-s, with similar recovery intervals, show that older muscle fatigues less than young $25,7,9,12$. However, where the duty cycle was changed so there was a shorter contraction / recovery interval such as in this study and in other reports ${ }^{13-15}$, there was no difference in the extent of fatigue in young and old subjects.

\section{Sex differences}

In this study, men and women had similar time to task failure when sustaining the contraction at 50\% MVC. Although this is in agreement with the work of Maughan et al42, a larger sample size might be needed in future studies to account for the considerable inter-individual variability. In studies that utilized a lower target force in the knee extensors, women were found to have a longer time to task failure than men ${ }^{43,42}$. Interestingly, where sex differences were reported in young subjects holding an isometric contraction of elbow flexors, they no longer existed in older men and 
women ${ }^{3}$. We have observed that the greater fatigue resistance of young women compared with men performing intermittent contractions continues into old age (Figure 6). We had previously attributed greater fatigue resistance during intermittent contractions to a slower muscle phenotype in young women compared to men ${ }^{16}$ arguing that this led to slower ATP turnover and thus slower fatigue. In the present study we again observed slower muscle in women compared with men, but another critical factor could be the difference in oxidative capacity, or the regeneration of ATP during the brief recovery interval between contractions. The fact that the difference in fatigue resistance between men and women during the intermittent contractions persists into old age indicates that the differences between men and women in rates of ATP use or its recovery also continue into old age.

\section{Limitations}

A limitation of this study into age- and sex-differences in muscle fatigue is that the models of muscle activation using a mix of electrical stimulation and voluntary contractions limits the extent to which direct correlations can be made between the results derived from the 2 types of fatigue test. Furthermore, the results from the single joint are not fully representative patterns of muscle activation in vivo, such as walking or stair climbing. In 'real-life' scenarios, fatigue is influenced by multiple systems that integrate human movement such as neural control, cardiorespiratory adjustments, motivation, perception of effort, and sensory feedback. When trying to understand the occurrence of fatigue during usual daily tasks it should be born in mind that, quantitatively, older muscles are smaller and weaker than young muscles and since, in general, body weight tends to increase with age, the older muscles will be working at a higher relative load. The tests of fatigability that we and most other groups have used 
have been standardized to the strength of the muscle, not to the tasks that they are required to perform in daily activities. Consequently, the commonly used tests of fatigability may underestimate the actual stress of daily activities on older people.

\section{Conclusion}

We conclude that older muscle is, in general, more resistant to fatigue than young when performing sustained isometric contractions of the knee extensors. However, following a series of 60 brief, intermittent isometric contractions young and old did not differ in the extent of fatigue. Significant sex differences were observed following the brief, intermittent contractions, where young and older women had better fatigue resistance compared with men, but men and women showed similar fatigue during a sustained isometric contraction. 


\section{ACKNOWLEDGEMENTS}

We wish to thank the volunteers for taking part in this study and acknowledge the funding provided by European Union FP7 MYOAGE (nr. 223576)

\section{Disclosures}

There are no conflicts of interest to declare.

\section{Author contributions}

All authors contributed to study conception, design, results interpretation and approved the final manuscript. JM \& TM additionally collected and analysed the data. 


\section{REFERENCES}

1. Bilodeau M, Erb MD, Nichols JM, Joiner KL, Weeks JB. Fatigue of elbow flexor muscles in younger and older adults. Muscle Nerve 2001;24(1):98-106.

2. Bilodeau M, Henderson TK, Nolta BE, Pursley PJ, Sandfort GL. Effect of aging on fatigue characteristics of elbow flexor muscles during sustained submaximal contraction. J Appl Physiol 2001;91(6):2654-2664.

3. Hunter SK, Critchlow A, Enoka RM. Influence of aging on sex differences in muscle fatigability. J Appl Physiol 2004;97(5):1723-1732.

4. Narici MV, Bordini M, Cerretelli P. Effect of aging on human adductor pollicis muscle function. J Appl Physiol 1991;71(4):1277-1281.

5. Griffith EE, Yoon T, Hunter SK. Age and load compliance alter time to task failure for a submaximal fatiguing contraction with the lower leg. J Appl Physiol (1985) 2010;108(6):1510-1519.

6. de Ruiter CJ, Goudsmit JF, Van Tricht JA, de Haan A. The isometric torque at which knee-extensor muscle reoxygenation stops. Med Sci Sports Exerc 2007;39(3):443-453.

7. Lanza IR, Russ DW, Kent-Braun JA. Age-related enhancement of fatigue resistance is evident in men during both isometric and dynamic tasks. J Appl Physiol 2004;97(3):967-975.

8. Kent-Braun JA, Ng AV, Doyle JW, Towse TF. Human skeletal muscle responses vary with age and gender during fatigue due to incremental isometric exercise. J Appl Physiol 2002;93(5):1813-1823.

9. Chung LH, Callahan DM, Kent-Braun JA. Age-related resistance to skeletal muscle fatigue is preserved during ischemia. J Appl Physiol 2007;103(5):1628-1635.

10. Ditor DS, Hicks AL. The effect of age and gender on the relative fatigability of the human adductor pollicis muscle. Can J Physiol Pharmacol 2000;78(10):781-790.

11. Rubinstein S, Kamen G. Decreases in motor unit firing rate during sustained maximal-effort contractions in young and older adults. J Electromyogr Kinesiol 2005;15(6):536-543.

12. Callahan DM, Foulis SA, Kent-Braun JA. Age-related fatigue resistance in the knee extensor muscles is specific to contraction mode. Muscle Nerve 2009;39(5):692702. 
13. Stackhouse SK, Stevens JE, Lee SC, Pearce KM, Snyder-Mackler L, Binder-Macleod SA. Maximum voluntary activation in nonfatigued and fatigued muscle of young and elderly individuals. Phys Ther 2001;81(5):1102-1109.

14. Allman BL, Rice CL. An age-related shift in the force-frequency relationship affects quadriceps fatigability in old adults. J Appl Physiol 2004;96(3):10261032.

15. Stevens JE, Binder-Macleod S, Snyder-Mackler L. Characterization of the human quadriceps muscle in active elders. Archives of physical medicine and rehabilitation 2001;82(7):973-978.

16. Wust RC, Morse CI, de Haan A, Jones DA, Degens H. Sex differences in contractile properties and fatigue resistance of human skeletal muscle. Experimental physiology 2008;93(7):843-850.

17. Mademli L, Arampatzis A. Effect of voluntary activation on age-related muscle fatigue resistance. Journal of biomechanics 2008;41(6):1229-1235.

18. Rutherford OM, Jones DA, Newham DJ. Clinical and experimental application of the percutaneous twitch superimposition technique for the study of human muscle activation. J Neurol Neurosurg Psychiatry 1986;49(11):1288-1291.

19. McPhee JS, Hogrel JY, Maier AB, Seppet E, Seynnes OR, Sipila S, et al. Physiological and functional evaluation of healthy young and older men and women: design of the European MyoAge study. Biogerontology 2013;May 31. [Epub ahead of print]:DOI:10.1007/s10522-10013-19434-10527.

20. Van Leeuwen DM, De Ruiter CJ, De Haan A. Effect of stimulation intensity on assessment of voluntary activation. Muscle Nerve 2012;45(6):841-848.

21. Katsiaras A, Newman AB, Kriska A, Brach J, Krishnaswami S, Feingold E, et al. Skeletal muscle fatigue, strength, and quality in the elderly: the Health ABC Study. J Appl Physiol 2005;99(1):210-216.

22. Degens H, Salmons S, Jarvis JC. Intramuscular pressure, force and blood flow in rabbit tibialis anterior muscles during single and repetitive contractions. European journal of applied physiology and occupational physiology 1998;78(1):13-19.

23. Lexell J, Taylor CC, Sjostrom M. What is the cause of the ageing atrophy? Total number, size and proportion of different fiber types studied in whole vastus lateralis muscle from 15- to 83-year-old men. J Neurol Sci 1988;84(2-3):275-294. 
24. Tevald MA, Foulis SA, Lanza IR, Kent-Braun JA. Lower energy cost of skeletal muscle contractions in older humans. Am J Physiol Regul Integr Comp Physiol 2010;298(3):R729-739.

25. Lanza IR, Larsen RG, Kent-Braun JA. Effects of old age on human skeletal muscle energetics during fatiguing contractions with and without blood flow. J Physiol 2007;583(Pt 3):1093-1105.

26. Green HJ. Cation pumps in skeletal muscle: potential role in muscle fatigue. Acta physiologica Scandinavica 1998;162(3):201-213.

27. Christie A, Snook EM, Kent-Braun JA. Systematic review and meta-analysis of skeletal muscle fatigue in old age. Med Sci Sports Exerc 2011;43(4):568-577.

28. Avin KG, Law LA. Age-related differences in muscle fatigue vary by contraction type: a meta-analysis. Phys Ther 2011;91(8):1153-1165.

29. Gregory CM, Bickel CS. Recruitment patterns in human skeletal muscle during electrical stimulation. Physical therapy 2005;85(4):358-364.

30. Lexell J, Taylor CC. Variability in muscle fibre areas in whole human quadriceps muscle: effects of increasing age. J Anat 1991;174:239-249.

31. Maffiuletti NA. Physiological and methodological considerations for the use of neuromuscular electrical stimulation. Eur J Appl Physiol 2011;110(2):223-234.

32. Houmard JA, Weidner ML, Gavigan KE, Tyndall GL, Hickey MS, Alshami A. Fiber type and citrate synthase activity in the human gastrocnemius and vastus lateralis with aging. J Appl Physiol (1985) 1998;85(4):1337-1341.

33. Conley KE, Jubrias SA, Esselman PC. Oxidative capacity and ageing in human muscle. The Journal of physiology 2000;526 Pt 1:203-210.

34. Crane JD, Devries MC, Safdar A, Hamadeh MJ, Tarnopolsky MA. The effect of aging on human skeletal muscle mitochondrial and intramyocellular lipid ultrastructure. J Gerontol A Biol Sci Med Sci 2010;65(2):119-128.

35. Safdar A, Hamadeh MJ, Kaczor JJ, Raha S, Debeer J, Tarnopolsky MA. Aberrant mitochondrial homeostasis in the skeletal muscle of sedentary older adults. PLoS One 2010;5(5):e10778.

36. Short KR, Bigelow ML, Kahl J, Singh R, Coenen-Schimke J, Raghavakaimal S, et al. Decline in skeletal muscle mitochondrial function with aging in humans. Proc Natl Acad Sci U S A 2005;102(15):5618-5623. 
37. Kaczor JJ, Ziolkowski W, Antosiewicz J, Hac S, Tarnopolsky MA, Popinigis J. The effect of aging on anaerobic and aerobic enzyme activities in human skeletal muscle. J Gerontol A Biol Sci Med Sci 2006;61(4):339-344.

38. Degens H. Age-related changes in the microcirculation of skeletal muscle. Advances in experimental medicine and biology 1998;454:343-348.

39. Fenn WO. A quantitative comparison between the energy liberated and the work performed by the isolated sartorius muscle of the frog. J Physiol 1923;58(23):175-203.

40. Barclay CJ, Constable JK, Gibbs CL. Energetics of fast- and slow-twitch muscles of the mouse. J Physiol 1993;472:61-80.

41. Newham DJ, Jones DA, Turner DL, McIntyre D. The metabolic costs of different types of contractile activity of the human adductor pollicis muscle. J Physiol 1995;488 ( Pt 3):815-819.

42. Maughan RJ, Harmon M, Leiper JB, Sale D, Delman A. Endurance capacity of untrained males and females in isometric and dynamic muscular contractions. Eur J Appl Physiol Occup Physiol 1986;55(4):395-400.

43. Clark BC, Collier SR, Manini TM, Ploutz-Snyder LL. Sex differences in muscle fatigability and activation patterns of the human quadriceps femoris. Eur J Appl Physiol 2005;94(1-2):196-206. 


\section{Figure Legends}

Figure 1: Example raw data from a sustained fatigue test in a young and older man. The test began with a stimulated doublet to approximately 30\% MVC and immediately after, the voluntary contraction began. Electrically-stimulated doublets were superimposed every 10 -s throughout the test, indicated by the arrows. The twitch-interpolation technique was used to assess the level of voluntary activation following each stimulus.

Figure 2: Torque-frequency relationship. Mean \pm SEM are shown for young (filled squares) and older subjects (open squares). Subplots show typical torque traces for a young (y) and older man (o) at each stimulation frequency. * indicates significant difference between age groups. No significant differences were seen between sexes.

Figure 3: Individual data points for sustained isometric contraction held at 50\% MVC until task failure. No significant difference was found between men (squares) and women (triangles), but old sustained the contraction for longer than young. *Indicates significant difference between young and old of $P<0.0005$.

Figure 4: Progression of peripheral muscle voluntary activation during the sustained contraction fatigue test in young (filled squares, solid trend line) and older subjects (open squares, dashed trend line).

Figure 5: Time to task failure during the sustained contraction held at 50\% MVC plotted as a function of MVC. Young men (filled squares); young women (filled triangles); older men (open squares) and older women (open triangles). 
Figure 6: Decline in torque during the intermittent isometric contraction fatigue test. A: young men (filled squares) and older men (open squares). B: Young women (filled triangles) and older women (open triangles). The protocol included 60 stimulated 30Hz contractions of 1 -s, each separated by a 1-s relaxation interval. Mean \pm SEM are shown. There was no significant age effect, but women fatigued significantly less than men. 


\section{Tables}

\begin{tabular}{|l|llll|}
\hline Variable & YM & YW & OM & OW \\
\hline Age & $22.4(3.1)$ & $22.3(1.9)$ & $68.9(4.4)$ & $71.1(3.4)$ \\
Height (m) & $1.81(0.07)$ & $1.69(0.03)$ & $1.74(0.07)$ & $1.58(0.06)$ \\
Body mass & $78.9(9.3)$ & $61.9(3.9)$ & $73.2(5.0)$ & $59.3(10.6)$ \\
BMI & $24.0(3.3)$ & $21.6(1.5)$ & $24.1(1.9)$ & $23.9(4.4)$ \\
\hline
\end{tabular}

Table 1: Subject characteristics. Mean (SD) in young men (YM), young women (YW), older men (OM), and older women (OW). 


\begin{tabular}{|c|c|c|c|c|}
\hline Variable & YM & YW & $\mathrm{OM}$ & OW \\
\hline $\operatorname{MVC}(\mathrm{Nm})^{* \dagger}$ & $286.7(12.0)$ & $175.2(6.4)$ & $167.5(10.8)$ & $101.2(6.2)$ \\
\hline$\%$ activation * & $89.6(1.5)$ & $92.9(1.1)$ & 86.9 (1.4) & 87.2 (2.9) \\
\hline $\begin{array}{l}\text { Normalized relaxation } \\
\text { rate }\left(\mathrm{s}^{-1}\right)^{*+}\end{array}$ & $16.8(0.9)$ & $14.0(0.8)$ & $12.9(0.7)$ & $12.1(0.5)$ \\
\hline $\begin{array}{l}1 / 2 \text { relaxation time } \\
(\mathrm{ms})^{* \dagger}\end{array}$ & $65.4(3.6)$ & $77.0(9.6)$ & $84.3(4.6)$ & 96.1 (5.9) \\
\hline
\end{tabular}

Table 2: Contractile characteristics of quadriceps femoris. Mean (SEM) in young men (YM), young women (YW), older men (OM), and older women (OW). MVC: maximal voluntary contraction; \% activation: percentage of muscle activated during MVC; Normalized relaxation rate and $1 / 2$ relaxation time have been normalized to peak torque. $1 / 2$ relaxation time is the time taken to relax from the final pulse of a $30 \mathrm{~Hz}$ electricallystimulated contraction to $50 \%$ peak torque. Data are mean (SEM). * indicates significant age difference; ${ }^{\dagger}$ indicates significant sex difference. 